\title{
Spin-Multislice Applied to the Electron Spin Interaction with Materials
}

Vincenzo Grillo ${ }^{1,2,3}$, Spencer Alexander ${ }^{3}$, Jan Rusz ${ }^{4}$, Alexander Edström ${ }^{4}$, Axel Lubk ${ }^{5}$, B J McMorran ${ }^{3}$, Ebrahim Karimi ${ }^{6}$

1. CNR-Istituto Nanoscienze, Centro S3, Via G. Campi 213/a, I-41125 Modena, Italy

2. CNR-IMEM Parco Area delle Scienze 37/A, I-43124 Parma, Italy

3. Department of Physics, University of Oregon, Eugene, Oregon, USA

4. Department of Physics and Astronomy, Uppsala University, Uppsala, Sweden

5. Triebenberg Laboratory, TU Dresden, Dresden, Germany.

6. Department of Physics, University of Ottawa, 25 Templeton, Ottawa, Ontario, K1N 6N5 Canada

The multislice method is one of the most efficient and flexible methods to solve the propagation problem of a wave in the paraxial approximation. It has lent itself to a number of extensions which incorporate back-scattering effects and thermal motion due to phonons. However, the inclusion of relativistic effects, and in particular of spin, is a recent achievement [1][2][3].

In extending the multislice method, both Pauli equation- and Dirac equation-based approaches have been pursued. The Pauli equation in particular is probably sufficient to deal with most of the measurable effects in electron microscopy, however particular care has to be taken in Lorentz boosting the field in the appropriate reference frame [2].

To date, the Pauli equation-based approach has been applied only to the interaction of electrons with the external field of a lens system. In particular, ref [2] proposed an extension of the Fourier Transform (FT) -based method that allows for the inclusion of the components of the magnetic potential field orthogonal to the propagation direction. While these terms are naturally included in real-space multislice simulations [3], their inclusion in FT multislice algorithm has required a strong change.

The extension of the formalism to include the interaction of electrons with the field inside of a material is more complicated. The main reason for this is that magnetic fields are typically extended over distances much larger than the lattice periodicity. We will therefore discuss a few possible approximations that can be used to describe this interaction, and especially the validity of a short-range cutoff that permits the incorporation of magnetic field effects on the atomic scales, where the field tends to be most intense.

Fig1 shows a few columns of Fe [001] which are magnetized in the horizontal direction, orthogonal to the propagation direction. This situation could only be experimentally produced in Lorentz mode, since the objective field would produce a very large field along the direction of propagation magnetizing the sample in that direction. It is nonetheless useful as a case study.

Fig 1a is an image of the exit wavefunction after interaction with the atomic electrostatic potential. Fig $1 b$ is an image of the phase effect due to the Lorentz force produced by atoms, which are modeled as dipoles. Fig $1 \mathrm{c}$ is the part of the wavefunction that has undergone a spin flip due to interaction with the atomic magnetic field. According to mechanisms highlighted in a previous work [4], the spin flip is associated with a change in the orbital angular momentum of the wavefunction.

Unfortunately, and, consistently with scalar multislice simulations, we find that Orbital Angular Momentum (OAM) is not conserved in the elastic interactions due to the non-circularly symmetric crystalline potential. A noticeable exception is the case when vortex states are coupled with the Bloch waves of lower transverse energy which feel a locally radially symmetric potential [5]. Fig 2 shows the simulated propagation of a very narrow vortex beam after interaction with a sample. Fig 2a is an image of the exit wavefunction of a probe concentrated on a Fe column. Figures $2 b$ and $c$ are images of the 
small fraction of the wavefunction that has undergone a spin flip. The difference between the two images depends upon the opposite spin-OAM projection of the initial state. We can see that the OAM of the final state is drastically different in the two cases.

\section{References:}

[1] Axel Rother, Kurt Scheerschmidt Ultramicroscopy 109 (2009) 154-160

[2] Vincenzo Grillo, Lorenzo Marrucci, Ebrahim Karimi, Riccardo Zanella and Enrico Santamato New journal Phys 15 (2013) 093026

[3] Alexander Edström, Jan Rusz, IT-16-P-3287, $18^{\text {th }}$ International Microscopy Congress 2014

[4] Ebrahim Karimi, Lorenzo Marrucci, Vincenzo Grillo, and Enrico Santamato Phys Rev Lett 108, $044801(2012)$

[5] Huolin L. Xin and Haimei Zheng Microsc. Microanal. 18 (2012), 711-719

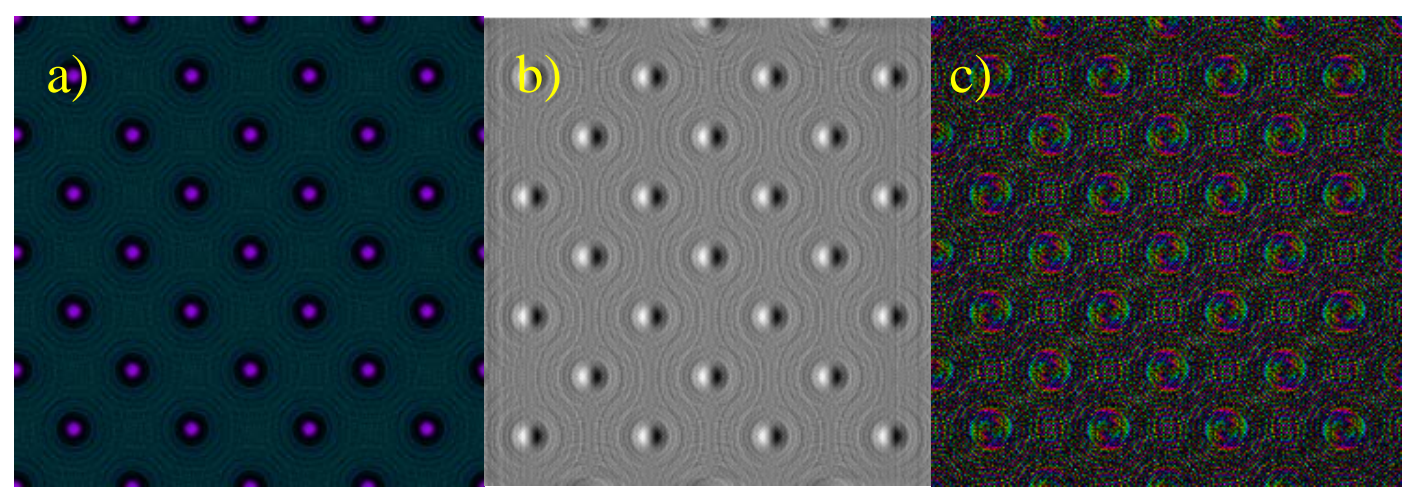

Figure 1. a) Exit wave after the interaction with the electrostatic potential of a Fe [100] cell. b) Lorentz phase effect introduced by the Fe atoms, which are modeled as dipoles. c) Wavefunction of the electrons that have undergone a spin flip during the interaction with the magnetic field. In both fig a and c, the intensity is proportional to the color brightness, and the phase is proportional to the hue.
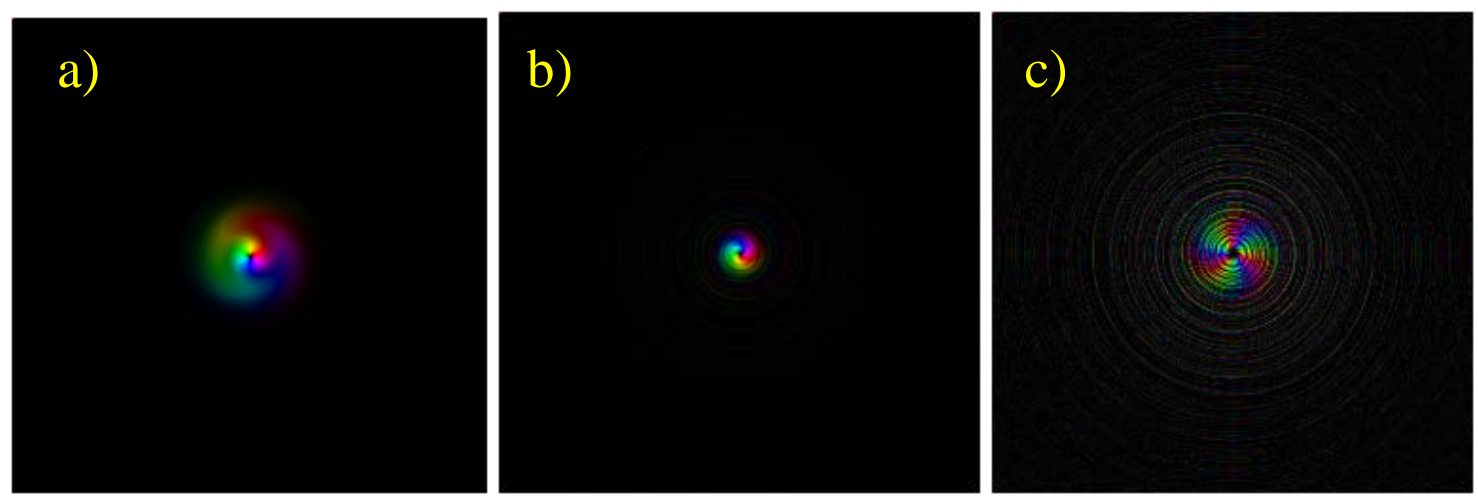

Figure 2. a) Exit wave after the interaction of a convergent probe with the electrostatic, magnetic and Pauli potential of a Fe [100] cell. b,c) Fraction of the exit wave that has undergone a spin- flip during the interaction in the case of (i) S.L $>0$, and (ii) S.L $<0$. The intensity is proportional to the color brightness, and the phase is proportional to the hue 